\title{
BEYOND THE VIRTUAL CRYSTAL APPROXIMATION IN DILUTED MAGNETIC SEMICONDUCTORS
}

\section{J. TWORZYDEO}

Institute of Theoretical Physics, Warsaw University, Hoża 69, 00-681 Warszawa, Poland

We present a new model approach which goes beyond the virtual crystal approximation and the molecular field approximation in the description of the spin splitting of band states in diluted magnetic semiconductors. The theory is formulated within a one-band effective mass approximation and explicitly takes into account a chemical and magnetic disorder caused by substitutional ions. The results confirm the apparent dependence of the $p-d$ exchange integral on $x$ in $\mathrm{Cd}_{1-x} \mathrm{Mn}_{x} \mathrm{~S}$ for small concentrations of $\mathrm{Mn}$ ions and we predict the asymmetric field and temperature dependence of the spin splitting in this compound.

PACS numbers: $71.70 . \mathrm{Gm}, 78.20 . \mathrm{Ls}, 71.55 . \mathrm{Gs}$

\section{Introduction}

An enormous progress has been already achieved in understanding the nature of magnetic interactions in diluted magnetic semiconductors (DMS) [1]. The magneto-optical experiments, which are particularly useful in this group of compounds, revealed the spectacular Zeeman splitting of extended states and allowed to determine precisely magnitudes of exchange integrals. The so far accepted theoretical interpretation of this splitting is based on the virtual crystal approximation (VCA) for an alloy disorder and the molecular field approximation (MFA) for an effective magnetization of substitutional magnetic ions [2].

The applicability of this approach should be limited and in fact some exceptions seem to exist. The more clear example of the departure from the VCA and the MFA [3] is perhaps the exceptionally large magnitude of the A-exciton field-induced spin splitting measured in $\mathrm{Cd}_{1-x} \mathrm{Mn}_{x} \mathrm{~S}$ for samples with small $x$. The exchange integrals for this compound inferred from experiments are concentration dependent and remain in disagreement with theoretical predictions. The authors in Ref. [3] have shown that the VCA indeed breaks down in the description of magneto-optical experiments in $\mathrm{Cd}_{1-x} \mathrm{Mn}_{x} \mathrm{~S}$ with $x \ll 1$ and that this breakdown is due to exceptionally large potential of $\mathrm{Mn}$ ions affecting the valence band. To 
avoid VCA they used a Wigner-Seitz approach introducing an artificial superlattice of $\mathrm{Mn}$ ions with the concentration dependent period.

In this paper we develop a model which accounts properly for both chemical disorder and strong attractive potential. We formulate the alloy approach in the framework of a standard theory [4] in the limit of small concentrations $x$ and then we generalize it for a magnetically disordered system.

\section{Solution of the model}

We start here with a simple description of a carrier in the one-band effective mass approximation subjected to random substitutional potentials and exchange interactions with magnetic ions. The exchange interaction is taken in the Heisenberg form and we use an approximation which neglects the processes of scattering a carrier with a spin-flip.

We solve the problem initially for a magnetic disorder suppressed by the saturating external magnetic field, that is putting the $\mathrm{Mn}$ ion spin operator eigenvalue $S_{n}=5 / 2$ for all impurity sites $n$. The effective dispersion relation for a particle in a random medium is given by its self-energy $\Sigma(k, E)$. We investigate here the limit of small impurity concentrations $x$ and in this approximation only corrections of the first order in $x$ are included [4] in the expression for $\Sigma$. Physically this approximation means that no interference between two successive scatterings of a carrier is allowed. We have after configurational averaging

$$
\Sigma(k, E)=N_{0} x t(k, E)
$$

where $t(k, E)=\langle k|t(E)| k\rangle$ is a diagonal element of the energy dependent scattering matrix $t$ for a one-impurity problem. The homogeneously distributed impurities do not mark out any specific length, therefore we do not expect any shift of the minimum of the dispersion relation away from $k=0$. The position of the band edge $E_{0}$ is then given by the equation

$$
E_{0}=\operatorname{Re} \Sigma\left(k=0, E_{0}\right) \text {. }
$$

We consider the solution of the general condition (2) for a Mn ion modeled by a square well potential $V(r)=V_{0} \Theta(b-r)$. In this case we solved in the analytic form the integral equation for the off-diagonal $t$-matrix elements. For weak impurity potentials our result reduces to the standard VCA expression for the band edge, but for potentials closer to the critical value for which the bound state occurs VCA is not valid and $t(k=0, E)$ depends in nontrivial way on $E$. This may lead to the nonlinear dependence of $E_{0}$ on $x$ despite the linear approximation for the self-energy (1).

The main advantage of our model is a possibility to include the effect of temperature and magnetic field on the spin splitting. For this purpose we allow each $\mathrm{Mn}$ ion spin to take one of the expected values $S_{z}=-5 / 2, \ldots, 5 / 2$ with the probability given by the Boltzmann distribution for independent spins. The magnitude of an impurity potential $V_{0}$ depends in our model on a magnetic ion spin value, thus we can construct the $S_{z}$-dependent $t$-matrix for scattering on potentials for different $S_{z}$. To determine the self-energy we perform the configurational averaging on a spin dependent $t$-matrix in the same way as in Eq. (1) and then 
we average it thermodynamically with the Boltzmann distribution. The position of the band edge is then given by Eq. (2) with the temperature and field dependent self-energy. The obtained result again reduces to the standard MFA and VCA expression for a weak impurity potential with the average spin value given by a Brillouin function.

\section{Results and discussion}

The procedure of adaptation of the model description to the case of $\mathrm{Cd}_{1-x} \mathrm{Mn}_{x} \mathrm{~S}$ was worked out in Ref. [3] and we follow it in our model calculations. The fenomenological parameters which specify the model are the exchange integral $\beta N_{0}$, the band-offset $W N_{0}$ for the valence band and the radius $b$ of the square well model potential. We find the parameter values which reproduce well the total A-exciton spin splitting at saturation reported by several groups [3] for samples with different concentrations $x$. There are however few experimental data and we get a good quality fit for a quite wide range of parameter values. The interpretation of these parameters and comparison with other predictions seems to be also limited by the inaccuracy of the square well model with an isotropic effective mass.

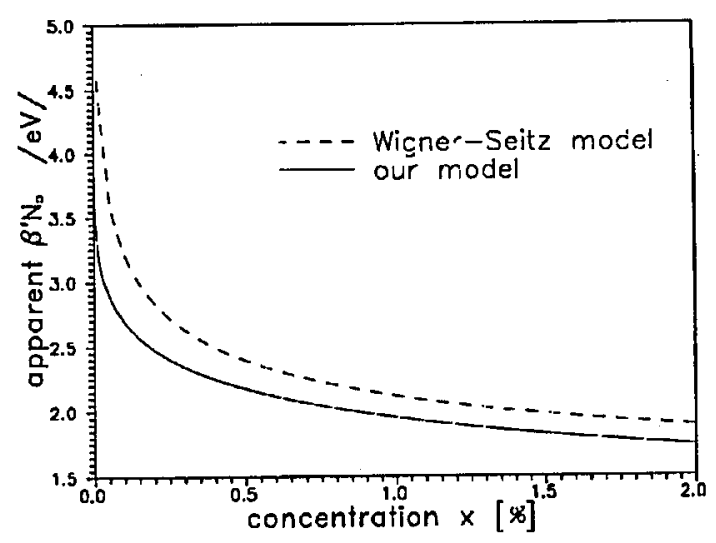

Fig. 1. Dependence of the apparent exchange integral $\beta^{\prime} N_{0}$ on $x$ in our model (solid line) and calculated in Ref. [3] (dashed line).

We illustrate a nonlinear dependence of the spin splitting on $x$ for one arbitrary chosen set of fitted parameters $\left(W N_{0}=-0.9 \mathrm{eV}, \beta N_{0}=-0.8 \mathrm{eV}, b=3 \AA\right)$ in Fig. 1. We have plotted there the apparent values of the exchange integral $\beta^{\prime} N_{0}$ equal to the calculated total spin splitting divided by the concentration $x$. The comparison with predictions of a Wigner-Seitz type intuitive model leads us to the conclusion that both models are qualitatively conformable.

We can also find the temperature and field dependence of the spin splitting using the fitted parameters. The result is plotted in Fig. 2, where the horizontal axis shows the magnetic field (or equivalently the inverse temperature) properly 


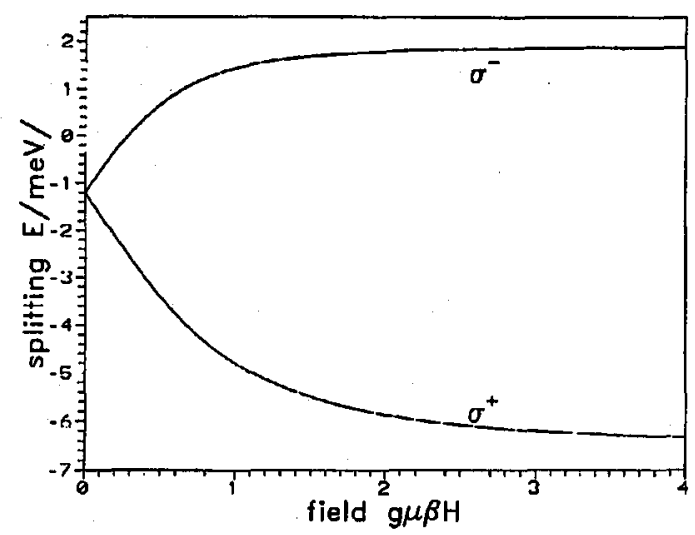

Fig. 2. The illustration of asymmetric spin splitting as a function of the external magnetic field for $x=0.1 \%$. The zero corresponds to the energy of exciton in pure CdS.

rescaled in dimensionless units. If the MFA and the VCA usual approach was valid, this dependence would be symmetric. Our model gives a clear asymmetry for the $\sigma^{-}, \sigma^{+}$exciton components, the stronger the lower concentration of magnetic impurities. This difference with a Brillouin type behavior is a new prediction and has not been so far considered theoretically.

The author would like to acknowledge Professor J. Blinowski for many useful discussions. The work was supported by the State Committee for Scientific Research (Republic of Poland), grant No. 2-P302-144-06.

\section{References}

[1] Diluted Magnetic Semiconductors, Eds. J.K. Furdyna, J. Kossut, in series Semiconductors and Semimetals, Vol. 25, Eds. R.K. Willardson, A.C. Beer, Academic, Boston 1988.

[2] J.A. Gaj, R. Planel, G. Fishman, Solid State Commun. 29, 435 (1979).

[3] C. Benoit à la Guillaume, D. Scalbert, T. Dietl, Phys. Rev. B 46, 9853 (1992) and references cited therein.

[4] H. Ehrenreich, L.M. Schwartz, in: Solid State Physics, Vol. 31, Eds. H. Ehrenreich, F. Seitz, D. Turnbull, Academic Press, New York 1976, p. 149. 